\title{
Spiking Patterns of Hodgkin-Huxley Model in Alzheimer's Disease: Effects Caused by Noise Current
}

\author{
Shruti Gupta \\ ITM University \\ ITM University, Sec-23 \\ Gurgaon
}

\author{
Jyotsna Singh \\ ITM University \\ ITM University, Sec-23 \\ Gurgaon
}

\begin{abstract}
In this paper we study the effect of noise on $\mathrm{HH}$ Model. Small-world, regular and random neural networks are the types of HH neuronal networks, which we study in spiking synchronization. External noise and Sub threshold stimulus are subjected to all the neurons. In neuronal membranes we study biophysically realistic $\mathrm{HH}$ neurons with intensity of intrinsic noise via voltage gated ion channels. There is an optimal strength of noise that is found in each of all the neuronal networks to induce maximal spiking synchronization. We further study that there is a range of synaptic conductance that is present in each of neuronal networks to induce the effect that an optimal strength maximizes the spiking synchronization of noise. Also, the effect diminishes if the synaptic conductance becomes small or large. The synaptic conductance decreases to maximize the effect as the connections between neurons increases.
\end{abstract}

\section{General Terms}

Hodgkin-Huxley model, neural networks, spiking synchronization, coupling strength

\section{Keywords}

External Current, noise current Strength, Gaussian white noise, Alzheimer's disease

\section{INTRODUCTION}

In nature the basic phenomenon is synchronization and has various applications in non linear systems (Huang $\mathrm{L}$ et al. 2004), linear systems (Dibner C et al. 2010), neural systems (Rossoni E et al. 2005) and so on. Interactions between coupled neurons are widely described by synchronization which demonstrates excitation [Rossoni E et al. 2005]. Neuronal oscillations are induced in the central nervous system (CNS) with the synchronization of neuronal excitations. In the brain (Wu X et al. 2007;Klimesch W et al. 2010; Arecchi F T 2004), for information binding, synchronization may be the mechanism. There are mainly two types of networks; small world (SW) (D.J. Watts et al. 1998) and scale-free (SF) network (A.-L. Barabasi, R. Albert, Science 286 1999). These networks have been widely used in capturing the characteristics of many real-world complex networks due to their potential. Small-world networks can support both local and distributed Information processing (D.S. Basset et al. 2006). A small average distance exists between vertices in SW network which shows clustering of high degree (Watts D J et al, 1998). SW networks behavior is possessed by networks like biological technological and social. Imaging-based biomarkers distinguish between Alzheimer's disease and healthy aging as an advantage of SW measures.
Small average distance and a high clustering coefficient are the two main characteristics of the SW networks which are displayed in the brain by many neuronal networks. Networks which are rich in theoretical meanings (Timme $\mathrm{M}$ et al. 2002; Gelenbe E et al. 2008; B“rgers C et al. 2003) and have centre for extensive investigation are the part of classic neuronal networks. These are regular and random neuronal networks. How activities of complex patterns are generated by neuronal circuitry is the major topic to be understood in theoretical and computational neuroscience. Regular and random networks are both dynamics characteristics of $\mathrm{HH}$ neurons which are SW network, are showed by Lago-Fernandez et al. (L.F. Lago-Fernandez et al. 2008). In the recent study we see that small-world HH networks (M. Ozer et al. 2008) are examined with noise-delayed decay phenomenon (NDD). In nervous systems of electrical behavior noises play an important role which is present in nervous system (Zeng $\mathrm{S} Y$ et al. 2007;H“nggi P 2002; Lindner B et al. 2004; Liu S B et al. 2012; Xu C et al. 2011; Liu S et al. 2011; Li L et al. 2011). In a single neuron spontaneous spiking (Zeng $S$ Y et al. 2007), stochastic resonance (H"nggi P 2002), and coherent resonance (Lindner B et al. 2004) can be induced through noise. During the last several years many interesting results have been obtained from synchronization of neural networks.

In neural networks, the concept of weak signal detection and information propagation is vital. Through stochastic resonance improvements in the representation of weak signals can be provided by noise (S.M. Bezrukov et al. 1995,J.E. Levin et al. 1996). With the increase in neuron number, node degree and connection density (Han F et al. 2009), the necessary coupling strength for phase and complete synchronization decreases. This was shown by a research on heterogeneous small-world neural networks. On synchronization, the network heterogeneity has an adverse effect (Shi $X$ et al. 2005). In some cases neural network coupling can be hybrid whereas it is generally uniform (Tang $Y$ et al. 2008). Synchronization behavior of neural network is affected by the properties of a single node in neural network which is an important factor, besides the network topology and coupling (Shi X et al. 2005). In this paper, in three different types of $\mathrm{HH}$ neuronal networks we study the spiking synchronization. It is found that in each of network there is the noise-induced maximal synchronization i.e. a range of synaptic conductance to cause the effect. The effect vanishes if the synaptic conductance is small or large. The synaptic conductance to maximize the effect decreases with the increase of the connections between neurons. Therefore, the noise induced in the Hodgkin-Huxley $(\mathrm{HH})$ neuronal networks is independent of certain types of neuronal networks. 


\section{MODEL AND METHODS}

Through the $\mathrm{HH}$ equation, the electrical behavior of an excitable neuron is described quantitatively. For the coupled $\mathrm{HH}$ neurons on the network, the time evolution of the membrane potential is given by: (Y. Gong et al. 2005, M. Ozer et al. 2008 and Yu Y et al. 2001)

$$
\begin{array}{rl}
C m\left(\frac{d V i}{d t}\right)=-g N & a m i^{3} * h i(V i-V N a)-g K * n i^{4} \\
& *(V i-V k)-g L(V i-V L) \\
& +\sum_{j} \varepsilon i j(V j-V i)+I i(e x t)
\end{array}
$$

Where $V i$ denotes the membrane potential of $i$-th neuron in the network, $C m=1 \mu \mathrm{F} / \mathrm{cm}^{2}$ is the specific capacitance of the neuronal membrane, whereas $g N a=120 \mathrm{mS} / \mathrm{cm}^{2}$ and $g K=36$ $\mathrm{mS} / \mathrm{cm}^{2}$ are the maximal sodium and potassium conductance, respectively. $g L=0.3 \mathrm{mS} / \mathrm{cm}^{2}$ is the leakage conductance which is assumed to be constant. Reversal potentials for sodium, potassium and leakage channels are $V N a=50 \mathrm{mV}$, $V k=-77 \mathrm{mV}$ and $V L=-54.4 \mathrm{mV}$ (MahmutOzer et al. 2009, Zhang Zheng-Zhen et al. 2012). $m i$ andhi are activation and inactivation variables for the sodium channeli. ni Is activation variable for potassium channel. Moreover, $\varepsilon i j$ denotes the coupling strength between neurons $i$ and $j$. Also, $I i(e x t)$ is the total external current received by the $i$-th neuron.

$$
\operatorname{Ii}(e x t)=I o+\xi i(t)
$$

As shown above, the external current $\operatorname{Ii}($ ext $)$ of the $i$-th neuron consists of external sub threshold stimulus $I o$, external noise current $\xi i(t)$. We consider the noise current $\xi i(t)$ as Gaussian white noise with (Zeng S et al. 2004).

$\langle\xi i(t)\rangle=0$,

$\left\langle\xi i(t) \xi i\left(t^{\prime}\right)\right\rangle=\sigma^{2} * \delta\left(t-t^{\prime}\right)$,

where $\sigma$ is the strength of noise current.

In $\mathrm{HH}$ model, in response to the membrane potential, activation and inactivation gating variablesmi, ni and hichanges over time (A.L. Hodgkin et al. 1952). HodgkinHuxley model follows the first-order differential equations. In voltage gated ion channels the stochastic dynamics can have important implications on the excitable behavior of neurons (E. Skaugen et al. 1979; J.T. Rubinstein et al. 1995; C.C. Chow et al. 1996; E. Schneidman et al. 1998; J.A. White et al. 2000; M. Ozer et al. 2005) only when the population of ion channels is finite. With different computational algorithm, different channel noise effects can be modeled (H. Mino et al. 2002).

In this paper, we will study that how external noise strength affect voltage in $\mathrm{HH}$ model. We plot graph between voltage and current with the help of main equation. Only external noise strength has maximum effect on the voltage.

\section{RESULTS AND DISCUSSIONS}

Io which is the external sub threshold stimulus is fixed to be $6.0 \mu \mathrm{A} / \mathrm{cm}^{2}$ in all the impersonations of this paper. Initially, in Small-world neuronal network we scrutinize the spiking synchronization. Adjusting the external noise current strength $\sigma=10 \mu \mathrm{A} / \mathrm{cm}^{2}$ and membrane potential i.e. $\mathrm{V}(1)=-60 \mathrm{mV}$.
We plot the graphs between current and voltage with respect to time.

From Figure 1 to Figure 3, we observe that voltage increases with increase in value of current. As shown in Figure 1, there is maximal spiking synchronization when $i$ is $2 \mu \mathrm{A}$. In Figure 1 and Figure 2, voltage increases linearly with the current. Figure 3 shows increasing voltage in non-linear fashion. From Figure 4 onwards, voltage decreases as well as increases nonlinearly as shown up to Figure 8.

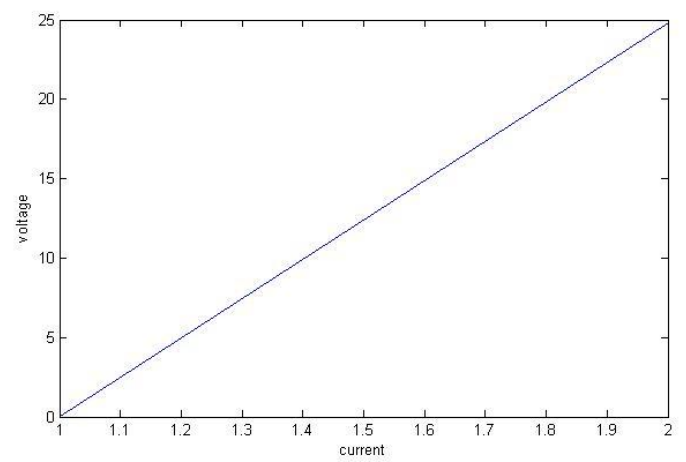

Figure 1: For $i=2 \mu \mathrm{A}$, we see that voltage increases linearly with increase in the current.

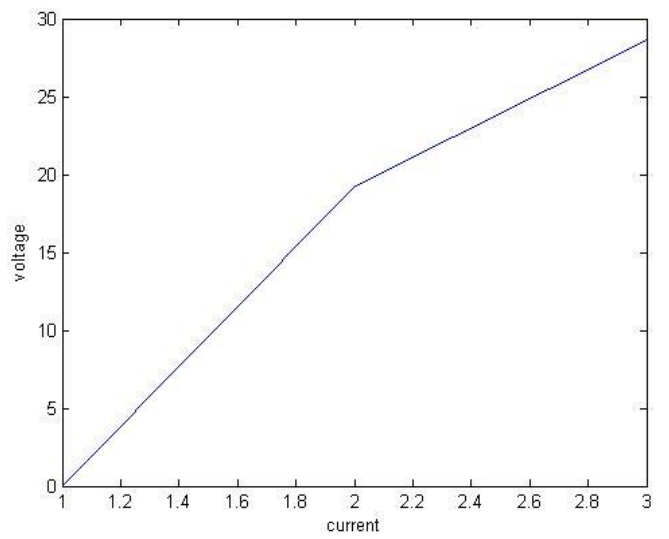

Figure 2: With $i=3 \mu \mathrm{A}$, shows that voltage increases with current linearly.

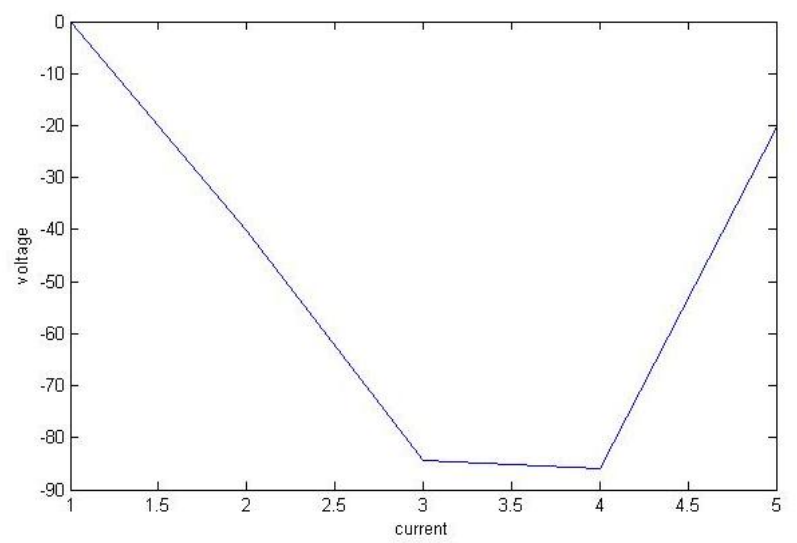

Figure 3: Taking $i=4 \mu \mathrm{A}$, voltage increases unhurriedly up to current point $2 \mu \mathrm{A}$, then remains constant and after wards increases swiftly. 


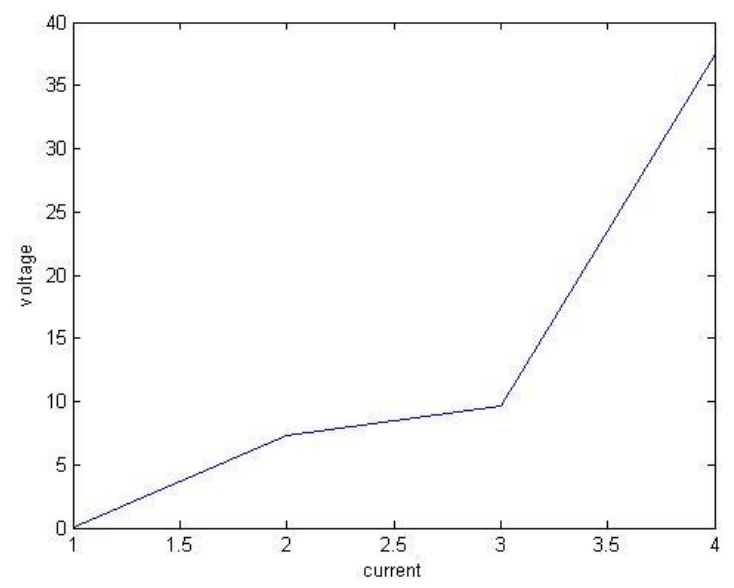

Figure 4: At $i=5 \mu \mathrm{A}$, voltage decreases rapidly and at point $3 \mu \mathrm{A}$ it starts increasing.

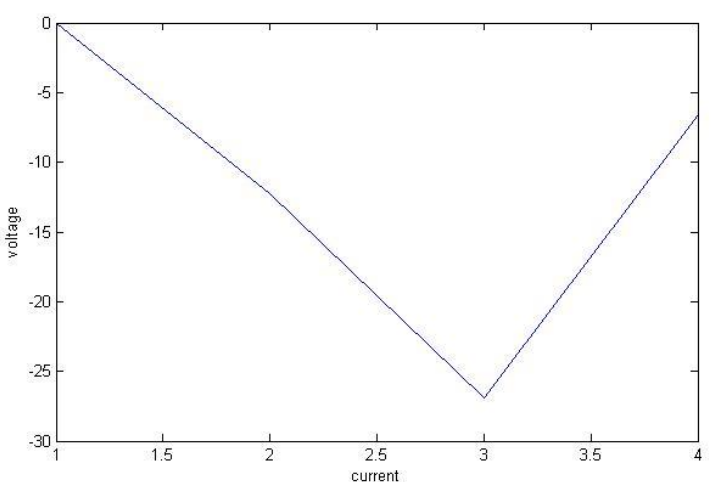

Figure 5: For $i=6 \mu \mathrm{A}$, voltage graph with current is in non-linear way.

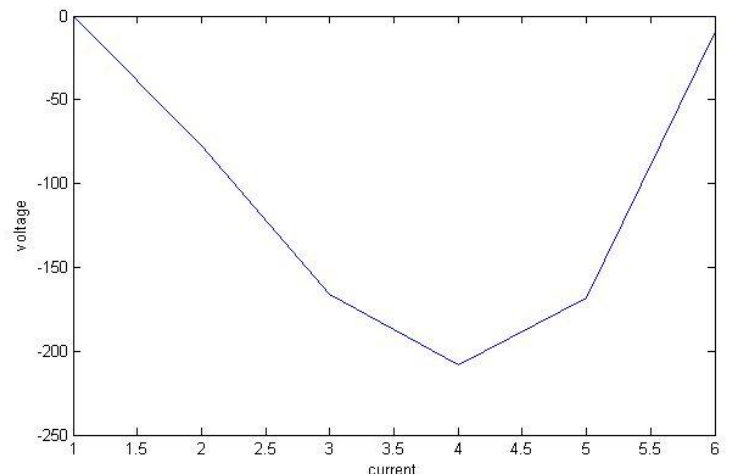

Figure 6: Now $i=7 \mu \mathrm{A}$, we see that voltage decreases till current value $4 \mu \mathrm{A}$ and then increases. Bents in the graph takes place while increasing and decreasing, also voltage remains same at point $3 \mu \mathrm{A}$ and $5 \mu \mathrm{A}$

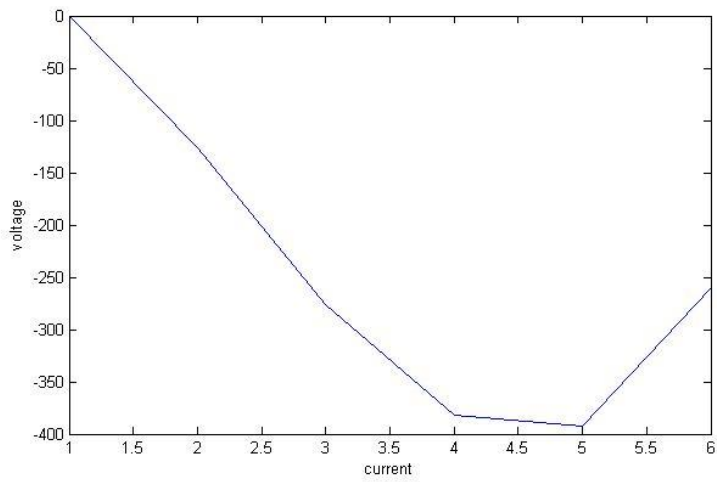

Figure 7: Taking $i=8 \mu \mathrm{A}$, shows that voltage continuously decreases till $-\mathbf{3 9 0} \mathrm{mV}$ and then slowly decreases till $\mathbf{- 4 0 0}$ $\mathbf{m V}$, afterwards it suddenly increases.

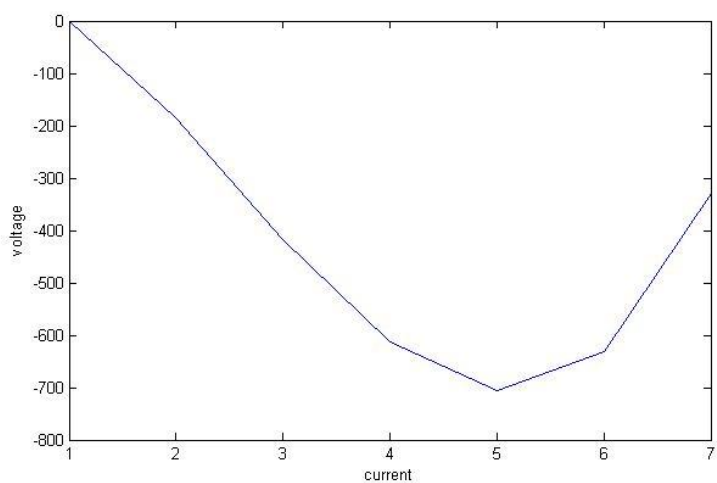

Figure 8: For value of $i=9 \mu \mathrm{A}$ and onwards graph remains almost same where it forms a curve.

In further study we take current a constant value i.e. $i=5 \mu \mathrm{A}$ and keep changing the external noise current strength i.e. $\sigma$. These changes in values of external noise current strength will have varying effects on voltage with respect to time.

Up to $\sigma$ value $12 \mu \mathrm{A} / \mathrm{cm}^{2}$, we can plot a graph similar to Figure 4. For value $\sigma=13 \mu \mathrm{A} / \mathrm{cm}^{2}$, we see that voltage decreases linearly with current as shown in Figure 9. In Figure 10 and Figure 11 , with $\sigma=14 \mu \mathrm{A} / \mathrm{cm}^{2}$ and $\sigma=15 \mu \mathrm{A} / \mathrm{cm}^{2}$ the values of voltage with current graph is non-linear. For values of $\sigma$ between $16 \mu \mathrm{A} / \mathrm{cm}^{2}$ and $19 \mu \mathrm{A} / \mathrm{cm}^{2}$ the graph is similar to Figure 12. For the values of $\sigma$ more than $20 \mu \mathrm{A} / \mathrm{cm}^{2}$, the graph almost looks as in Figure 13, which is again non-linear.

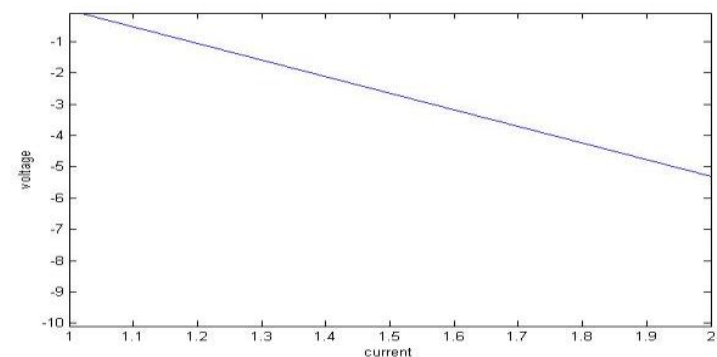

Figure 9: For value $\sigma=13 \mu \mathrm{A} / \mathrm{cm}^{2}$, the voltage decreases linearly. 


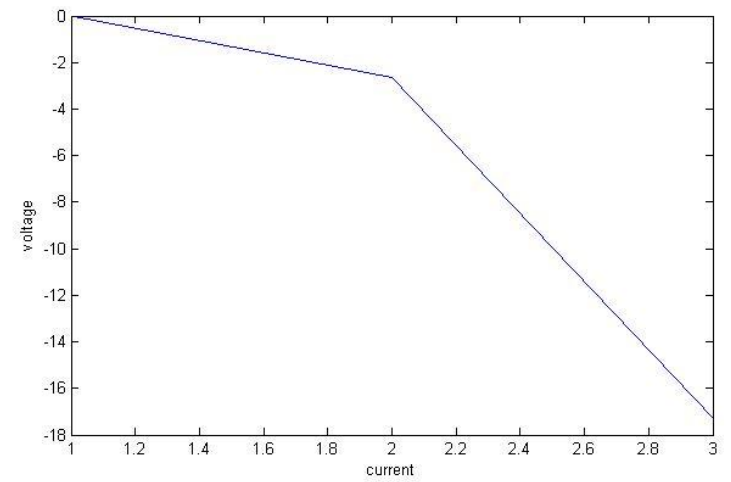

Figure 10: For value $\sigma=14 \mu \mathrm{A} / \mathrm{cm}^{2}$, the Voltage decreases slowly to current $2 \mu \mathrm{A}$, then it rapidly decreases.

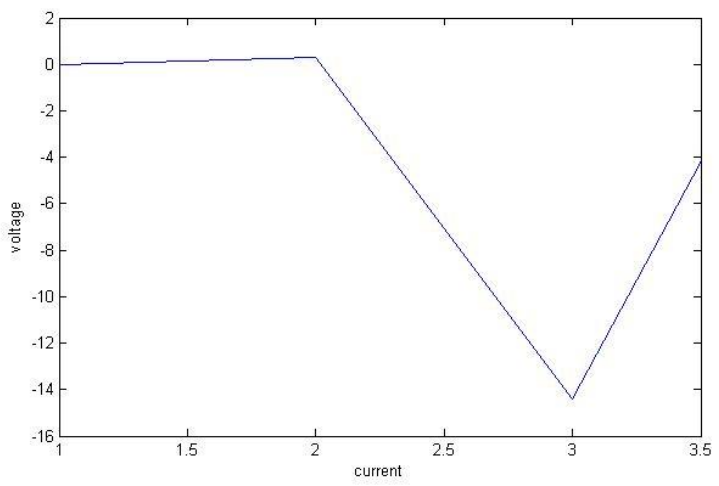

Figure 11: For value $\sigma=15 \mu \mathrm{A} / \mathrm{cm}^{2}$, the voltage remains constant after which it decreases and reaches $3 \mu \mathrm{A}$ and then increases.

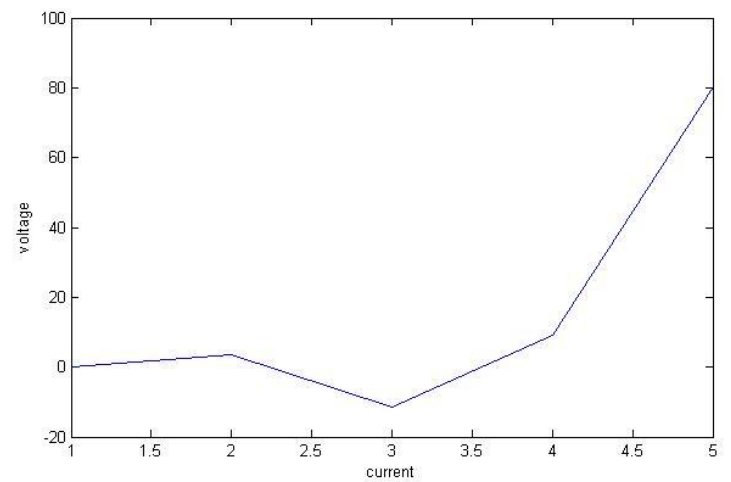

Figure 12: For value $\sigma=16 \mu \mathrm{A} / \mathrm{cm}^{2}$ to $19 \mu \mathrm{A} / \mathrm{cm}^{2}$, voltage keeps increasing and decreasing gradually.

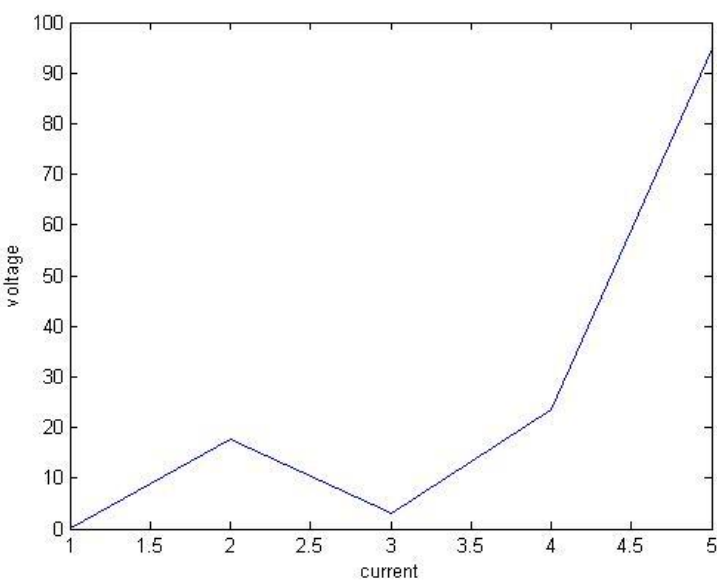

Figure 13: For values of $\sigma=20 \mu \mathrm{A} / \mathrm{cm}^{2}$ onwards, graph is almost same.

\section{CONCLUSION}

The small-world, random and the regular neuronal networks are the three different types of neuronal networks, whose spiking synchronization we studied. To induce neuronal oscillations and information binding (Ward L M 2003; Melloni L et al. 2007; Wu X et al. 2007; Klimesch W et al. 2010; Arecchi F T 2004), synchronization is a main harmony. In neuronal system, synchronization is an important phenomenon. Sub threshold stimulus and external noise are subject to neurons in the network. In all the neuronal networks, to cause the maximal spiking synchronization there is an optimal strength of noise. It is shown that to induce the effect i.e. the induced maximal synchronization, there is a range of synaptic conductance. The effect will be considerable only when the strength of synaptic conductance is a moderate value. The effect vanishes if the synaptic conductance is small or large. With the increase in connection probability between neurons as the optimal synaptic conductance maximizes, the effect decreases. In the neuronal networks, it is shown that general occurrence is noise-induced maximal synchronization.

The spiking synchronization of neuronal networks is better understood with the help of our research results. In this paper, we have shown in $\mathrm{HH}$ equation that how voltage changes with change in current and keeping all other parameters value constant. Also, we have shown that external noise current alone have a great effect on the voltage only for values of strength between $13 \mu \mathrm{A} / \mathrm{cm}^{2}$ to $19 \mu \mathrm{A} / \mathrm{cm}^{2}$ i.e. keeping current and all other parameters value constant, we see that there is great fluctuation in the graph.

\section{ACKNOWLEDGEMENT}

I would sincerely like to thank Mrs. Jyotsna Singh for her help and guidance.

\section{REFERENCES}

[1] Huang, L., Feng, R., Wang, M.,(2004).Synchronization of chaotic systems via nonlinear control.Physical Letter A, 320, 271-275.

[2] Dibner, C., Schibler, U., Albrecht, U.,(2010).The mammalian circadian timing system: organization and coordination of central and peripheral clocks.Annual Review of Physiology, 72, 517-549. 
[3] Rossoni, E., Chen, Y., Ding, M.,Feng, J.,(2005). Stability of synchronous oscillations in a system of Hodgkinhuxley neuron with delayed diffusive and pulsed coupling. Physical Review E 71, 061904.

[4] Ward, L. M.,(2003).Trends in Cognitive Sciences.Journal of Elsevier, Vol. 7, 553.

[5] Melloni. L., Molina. C., Pena.M., Torres. D., Singer.W., Rodriguez.E.,(2007).Synchronization of neuronal activity across cortical areas correlates with conscious perceptron.Neuroscience, 27(11), 2858-2868.

[6] Wu, X., Chen, X., Li, Z., Han, S., Zhang, D.,(2007). Binding of verbal and spatial information in human working memory involves large-scale neural synchronization at theta frequency. NeuroImage, 35, 1654-1662.

[7] Klimesch, W., Freunberger, R.,Sauseng, P.,(2010). Oscillatory behaviour of process binding in memory.NeuroscienceBiobehavioral Review, 34, 10021014.

[8] Arecchi, F. T.,(2004). Chaotic neuron dynamics, synchronization and feature binding.Physica A, 338, 218237.

[9] Watts, D, J., Strogatz, S, H., (1998). Collective dynamics of 'small-world' networks.Nature 393, 440-442.

[10] Barabasi, A, L., Albert, R., (1999). Emergence of scaling in random networks.Science, 286, 509-512.

[11] Basset,D,S., Bullmore, E., (2006). Small world brain networks.The Neuroscientist, 12, 512-523.

[12] Lago-Fernandez, L, F., Huerta, R., Corbacho, F., Siguenza, J, A., (2000).Fast response and temporal coherent oscillations in Small-world networks. Physical Review Letters, 84, 2758.

[13] Timme, M., Wolf, F., Geisel, T.,(2002). Computation byswitching in complex network of states.Physical Review Letter, 89, 258701.

[14] Gelenbe, E.,Timotheou, S., (2008). Random neural networks with synchronized interactions.Neural Computation, 20, 2308-2324.

[15] Borgers, C.,Kopell, N.,(2003). Synchronization in networks of excitatory and inhibitory neurons with spare, random connectivity.Neural Computation, 15, 509-538.

[16] Zeng, S. Y., Tang, Y., Jung, P., (2007). Physical Review E, 76, 011905 .

[17] Hanggi, P. (2002). Chem. Phys. Chem., 3, 285-290

[18] Lindner, B., Garcia-Ojalvo, J., Neiman, A.,SchimanskyGeier, L.,(2004). Effects of noise in excitable systems. Physical Reports, 392, 321-424.

[19] Liu S B, Wu Y, Hao Z W, Li Y J and Jia N (2012).Acta Phys. Sin. 61020503 (in Chinese)

[20] Xu, C., Kang, Y. M.(2011).Acta Phys. Sin.60, 108701 (in Chinese)

[21] Liu, S. J., Wang, Q., Liu, B., Yan, S. W.,Fumihiko, S.(2011). Noise transmission and delay induced stochastic oscillations in biochemical network motifs. Chinese Physics B, 20, 128703
[22] Li, L., Jin, Z. L., Li, B.,(2011).Acta Phys. Sin. 60, 048703 (in Chinese)

[23] Ozer, M., Uzuntarla, M., (2008). Effects of the network structure and coupling strength on the noise-induced response delay of a neuronal network. Physical letter A, 372, 4603-4609.

[24] Bezrukov, S. M., Vodyanov, I. (1995). Noise-induced enhancement of signal transduction across voltagedependent ion channels, Nature 378(6555), 362-364.

[25] Levin, J. E., Miller, J. P., (1996). Broadband neural encoding in the cricket cercal sensory system enhanced by stochastic resonance.Nature 380(6750), 165-168.

[26] Gong, Y., Wang, M., Zhou, Z., Xin, H. (2005) Chem. Phys. Chem. 6, 1042.

[27] Ozer, M., Uzuntarla, M., Perc, M., Graham, L. J. (2009). Spike latency and jitter of neuronal membrane patches with stochastic Hodgkin-Huxley channels. Physical Letter A, 261, 83-92.

[28] Han, F., Lu, Q. S., Wiercigroch, M.,Ji, Q. B.(2009). Complete and phase synchronization in a heterogeneous small-world neuronal network. Chinese Physics B, 18, 482 .

[29] Tang, Y.,Zhong, H., Fang, J.(2008). Synchronization of stochastically hybrid coupled neural networks with coupling discrete and distributed time-varying delays.Chinese Physics B, 17, 4080 .

[30] Shi, X., Lu, Q.(2005). Coherence resonance and synchronization of Hindmarsh-Rose neurons with noise.Chinese Physics, 14(06), 77.

[31] Yu, Y., Wang, W., Wang, J., Liu, F. (2001). Resonanceenhanced signal detection and transduction in the Hodgkin-Huxley neuronal systems. Phys. Rev. E 63, 021907.

[32] Ozer, M., Perc, M.,Uzuntarla,M. (2009). Stochastic resonance on Newman-Watts networks of HodgkinHuxley neurons with local periodic driving. Physical Letter A 373, 964-968.

[33] Zheng-Zhen, Z., Shang-You, Z., Wen-Yan, T., Jin-Lin, H., Shao-Wen, Z., Wei-Lian, N., Yi, Q., el al. (2012). Spiking synchronization regulated by noise in three types of Hodgkin-Huxley neuronal networks.Chinese Physics $B$, Vol. 21, No. 10108701 .

[34] Zeng, S., Jung, P.,(2004). Spiking synchronization of ion channel clusters on an axon.Physical Review E 70, 011903 .

[35] Hodgkin, A. L., Huxley, A. F. (1952). A quantitative description of membrane current and its application to conduction and excitation in nerve.J. Physiol. (London) $117,500-544$

[36] Skaugen, E., Walloe, L.,(1979). Firing behaviour in a stochastic nerve membrane model based upon the Hodgkin-Huxley equations. Acta Physiol. Scand. 107(4), 343-363.

[37] Rubinstein, J. T., (1995). Threshold fluctuations in an Nsodium channel model of the node of Ranvier. Biophysical, 68(3), 779-785. 
[38] Chow, C. C., White, J. A., (1996). Spontaneous action potentials due to channel fluctuations.Biophys. J. 71(6), 3013-3021.

[39] Schneidman, E., Freedman, B.,Segev, I., (1998).Ion Channel Stochasticity May Be Critical in Determining the Reliability and Precision of Spike Timing. Neural Comput. 10, 1679-1703.

[40] White, J. A., Rubinstein, J. T., Kay, A. R. (2000). Channel noise in neurons. Trends Neurosci. 23(3), 131137.
[41] Ozer, M.,Ekmekci, N. H., (2005). Phys. Lett. A338 150.

[42] Mino, H., Rubinstein, J. T., White, J. A. (2002). Comparison of algorithms for the simulation of action potentials with stochastic sodium channels.Ann. Biomed. Eng. 30(4), 578-587. 